\title{
BSM search in rare muon decay: the MEG II experiment
}

\section{Kei leki*on behalf of the MEG II collaboration}

The University of Tokyo,

7-3-1 Hongo, Bunkyo-ku, Tokyo, Japan

E-mail: kei.ieki@psi.ch

Lepton flavor violating decay of muon is one of the best probes for physics beyond the Standard Model (BSM). A golden channel, $\mu \rightarrow e \gamma$, will be searched by the MEG II experiment. MEG II aims to search $\mu \rightarrow e \gamma$ with ten times better branching ratio sensitivity than MEG, $6 \times 10^{-14}$ ( $90 \%$ C.L.). Many well motivated BSM theories predict sizable branching ratio of this decay (e.g. $O\left(10^{-14}\right)$ in SUSY-seesaw), which is within the reach of MEG II. MEG II utilizes world's highest intensity continuous $\mu^{+}$beam $\left(7 \times 10^{7}\right.$ muons/s) at Paul Scherrer Institute (PSI). In order to cope with extremely high rate accidental background of $e^{+}$and $\gamma$, all the detectors are upgraded from MEG. Detectors are installed and commissioned with muon beam at PSI. We present the status and prospect of the MEG II experiment.

40th International Conference on High Energy physics - ICHEP2020

July 28 - August 6, 2020

Prague, Czech Republic (virtual meeting)

${ }^{*}$ Speaker 


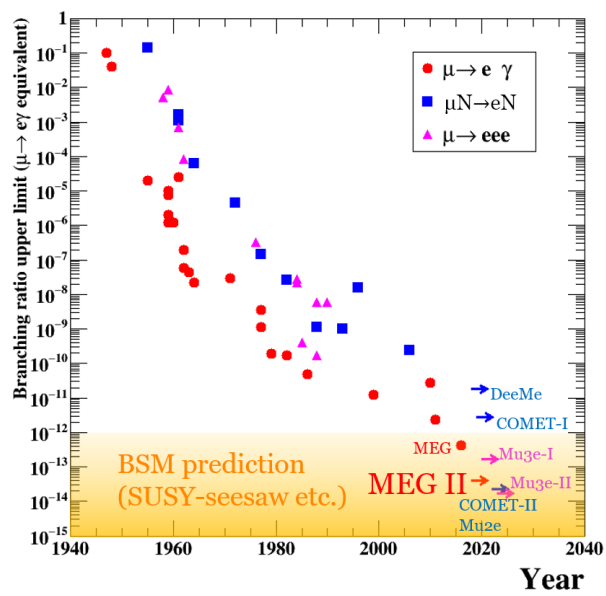

Figure 1: History of the branching ratio upper limits of rare muon decays.

\section{Muon as a probe of new physics}

Sign of BSM has been searched for by many experiments, but there has been no evidence of BSM discovered so far. Rare muon decays are one of the best probes of BSM. Complementary to high energy frontier experiments, rare muon decay is sensitive to BSM physics at $O(10) \mathrm{TeV}$ energy scale and beyond. MEG II experiment searches for the $\mu \rightarrow e \gamma$ decay, which will be, if observed, clear evidence of BSM physics[1].

Figure 1 shows the history of the branching ratio upper limits of three different types of lepton flavor violating muon decay modes: $\mu \rightarrow e \gamma, \mu \rightarrow e e e$ and $\mu N \rightarrow e N$. These three modes are known as "golden modes", and $\mu \rightarrow e \gamma$ has been the leading mode of these decays. While $\mu \rightarrow e \gamma$ is most sensitive to the photonic dipole interaction, the branching ratio of $\mu \rightarrow e e e$ and $\mu N \rightarrow e N$ becomes large if the contribution of contact interaction becomes large. Therefore, these three decay modes are sensitive to different types of new physics.

As shown in Fig. 1, current upper limits on the rare muon decay modes are already within the region predicted by the BSM physics such as SUSY-seesaw model. There are several new generation experiments which will search for those decay modes with improved sensitivity, utilizing high-intensity muon beams which are already available or being developed. Among those new generation experiments, MEG II is expected to start data taking first. The overview and the current status of the experiment is described in the following.

\section{MEG II experiment}

MEG II aims to search for the $\mu \rightarrow e \gamma$ decay with $\sim 6 \times 10^{-14}$ branching ratio upper limit sensitivity, which is ten times better than MEG. Major upgrades from MEG are as follows: a) Increase of muon stopping rate on target by factor of $2.3\left(7 \times 10^{7}\right.$ muon/sec.), b) Improvement of all detector resolutions by factor 2 , and increase of $e^{+}$detection efficiency by factor 2 .

PSI $590 \mathrm{MeV}$ proton cyclotron provides high-intensity proton beam at current up to $2 \mathrm{~mA}$. MEG II utilizes $\sim 28 \mathrm{MeV} / \mathrm{c}$ positive muon beam from the decay of secondary pions which stopped near the surface of the production target. Muon beam stops at a thin scintillating target at the center 


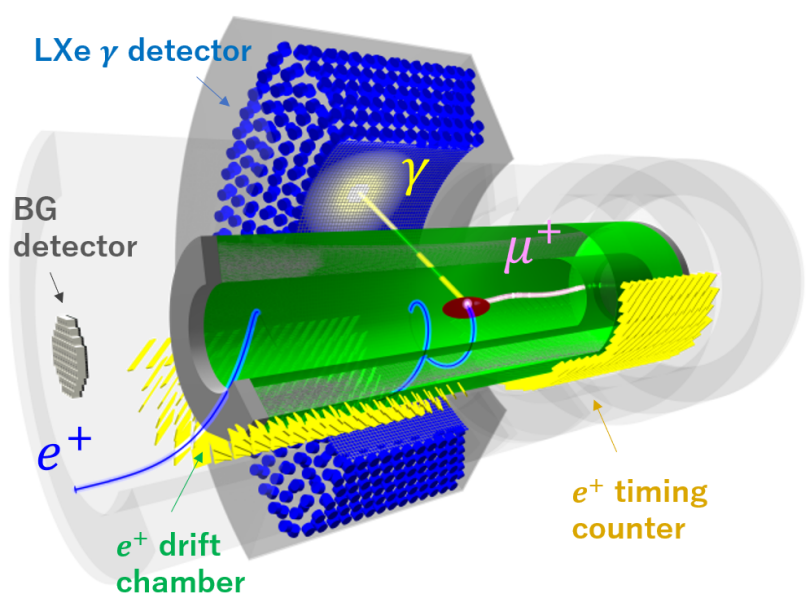

Figure 2: Overview of the MEG II detector apparatus

of the MEG II apparatus, and the $e^{+}$and $\gamma$ from the decay of muons are detected by the positron spectrometer and the liquid xenon gamma detector (Fig. 2).

The sensitivity of MEG was limited by the accidental backgrounds, namely the accidental coincidence of $e^{+}$and $\gamma$. Positrons are produced in Michel decay of muon $\mu \rightarrow e v \nu$, and $\gamma$ are produced in radiative muon decay $\mu \rightarrow e v v \gamma$ or by annihilation of $e^{+}$in flight. On the other hand, $\mu \rightarrow e \gamma$ signal is a simple two-body decay, with the energy of $e^{+}$and $\gamma$ equivalent to half of the muon mass. Therefore, we use following observables in the $\mu \rightarrow e \gamma$ search: energy of $e^{+}\left(E_{e}\right)$ and $\gamma\left(E_{\gamma}\right)$, time difference $\left(T_{e \gamma}\right)$ and opening angle $\left(\theta_{e \gamma}\right)$. Number of accidental background events $\left(N_{B G}\right)$ depends on the uncertainties of these observables:

$$
N_{B G} \propto R_{\mu}^{2} \times \Delta E_{\gamma}^{2} \times \Delta E_{e} \times \Delta \theta_{e \gamma}^{2} \times \Delta T_{e \gamma} \times T,
$$

where $\Delta E_{\gamma}, \Delta E_{e}, \Delta \theta_{e \gamma}$ and $\Delta T_{e \gamma}$ are the uncertainties of each observables, $R_{\mu}$ is the muon stopping rate, $T$ is the data taking time. Therefore in MEG II, improvement of the detector resolution is crucial for achieving the sensitivity goal.

All the detectors in MEG II are upgraded, and they are being commissioned with muon beam. In the following sections, we describe the design of the detectors and the status of the commissioning.

\section{$2.1 e^{+}$spectrometer}

The $e^{+}$spectrometer consists of a cylindrical drift chamber (CDCH, Fig. 3) and a timing counter (TC, Fig. 4).

\subsubsection{Cylindrical drift chamber}

$\mathrm{CDCH}$ is $1.91 \mathrm{~m}$ long cylinder, with $17 \mathrm{~cm}$ inner radius and $29 \mathrm{~cm}$ outer radius. It is installed inside the COBRA magnet, which is reused from MEG. A gradient magnetic field of COBRA is designed so that the bending radius of $e^{+}$becomes independent of the emission zenith angle. The background low momentum $e^{+}$from muon Michel decay does not hit the detector. In the MEG 


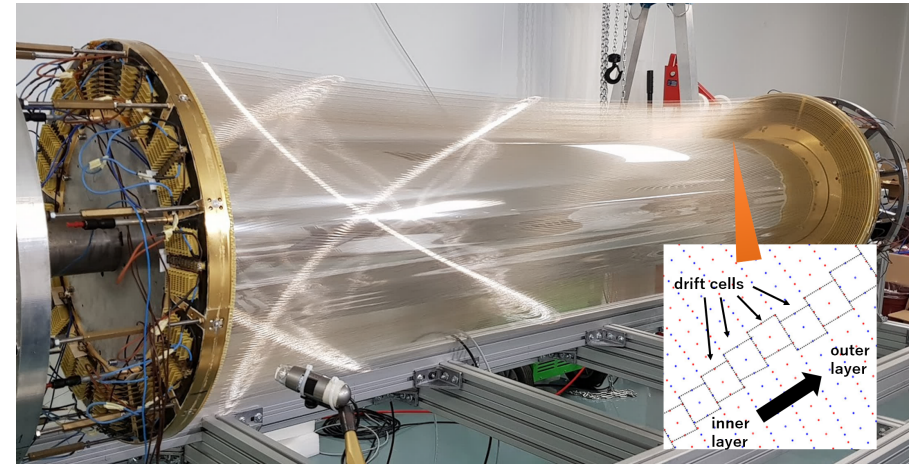

Figure 3: The cylindrical drift chamber

drift chamber, positron tracking efficiency was bad due to the readout electronics placed in between the chamber and the timing counter. The efficiency is expected to be doubled in MEG II because the electronics are moved away to the end of the long cylindrical volume.

$\mathrm{CDCH}$ is designed to have extremely low mass because the resolutions are limited by the multiple scattering. Gas mixture is based on Helium (He and iso-butane in 90:10). There are $\sim 12000$ wires used, among which $\sim 10000$ are the cathode wires and 1728 are the anode wires. Cathode wires are made of 40 or $50 \mu m$ thick Ag-plated aluminum and the anode wires are made of $20 \mu \mathrm{m}$ thick Au-plated tungsten. The total radiation length per track is $1.58 \times 10^{-3} X_{0}$, while it was $2.0 \times 10^{-3} X_{0}$ in MEG.

There are nine layers of anode wires with each layer having alternating signs of stereo angles (6-8.5 degrees). Each anode wire is surrounded by cathode wires to form a drift cell. The shape of the drift cells is approximately square, with the size ranging from 6 to $9 \mathrm{~mm}$. Single hit resolution expected from the prototype is $\sim 110 \mu \mathrm{m}$, while it was $\sim 200 \mu \mathrm{m}$ in MEG. Thanks to the improved transparency, better spacial resolution and the increased number of hits, resolution of momentum and emission angle are expected to be improved from MEG by a factor of 2 .

\subsubsection{Timing counter}

Hit timing of $e^{+}$is measured by the timing counter (TC). TC consists of 512 plastic scintillator counters, 256 at upstream and 256 at downstream (Fig. 2 and 4). Each of the counters is made of a fast plastic scintillator (BC-422, Saint Gobain). The width of each counter is $120 \mathrm{~mm}$, the thickness is $5 \mathrm{~mm}$, and the height is $40 \mathrm{~mm}$ or $50 \mathrm{~mm}$, depending on the position along the beam axis.

To achieve good time resolution of each counter, it is important to collect as much scintillation light as possible. Six SiPMs (AdvainSiD ASD-NUV3S-P-High-Gain) are attached on each side for scintillation light readout. Those SiPMs are connected in series to reduce the number of readout channels. A series connection is better than a parallel connection because the total capacitance becomes smaller and the pulse shape becomes sharp. With this configuration, $\sim 90$ psec time resolution is obtained for single counter.

The important concept of TC is to have multiple hits per one $e^{+}$track. Compared to the MEG timing counter which was composed of 30 scintillation bars, the MEG II TC is highly segmented. When there is $N_{\text {hit }}$ counter hits, the effect of the single counter resolution and the electronics jitter reduces by $1 / \sqrt{N_{\text {hit }}}$. On average, $N=9.3$ hits per signal $e^{+}$is expected, and the total time resolution 


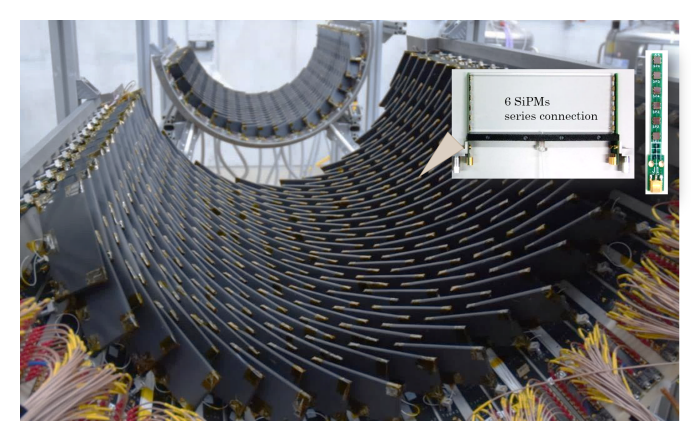

Figure 4: The timing counter

of $\sim 30 \mathrm{ps}$ can be achieved. The time resolution of $31 \mathrm{psec}$ at $N_{h i t}=9$ is already achieved in the commissioning run with $e^{+}$from the Michel decay of muon.

\subsection{Liquid xenon $\gamma$ detector}

MEG II utilizes $>900 l$ liquid xenon detector to measure the energy, time and position of $\gamma$ (Fig. 5). Liquid xenon scintillator has the following advantages 1) High scintillation yield, 2) High stopping power (radiation length is $2.77 \mathrm{~cm}$ ), 3) Fast response (45 ns time constant for $\gamma$ ) and 4) Uniform response (as a liquid). In MEG II, we reuse the liquid xenon and the cryostat from MEG.

The main upgrade of the detector is the replacement of the 216 PMTs (2-inch tubes) at the $\gamma$ entrance face to $4092 \mathrm{SiPMs}\left(12 \times 12 \mathrm{~mm}^{2}\right)$. With the improved granularity, position resolution is expected to improve by a factor of 2 for the events in which the $\gamma$ is converted near the entrance face (depth $<3 \mathrm{~cm}$ ). The energy resolution may also improve because of the improved uniformity of the area covered by photo-sensors.

A new type of SiPMs (Hamamatsu Photonics K.K., S10943-4372) is newly developed. It is sensitive to the scintillation light of xenon at vacuum ultraviolet wavelength $(\sim 175 \mathrm{~nm})$. Each SiPM has four $6 \times 6 \mathrm{~mm}^{2}$ chips, and the signal is read out as one channel by series connection inside PCB.

Improvement of position resolution is confirmed in commissioning run with $17.6 \mathrm{MeV} \gamma$ from ${ }^{7} \mathrm{Li}(\mathrm{p}, \gamma){ }^{8} \mathrm{Be}$ reaction. The resolution was measured to be $\sim 2.5 \mathrm{~mm}$ for $\gamma \mathrm{s}$ converted in the shallow region $(<2 \mathrm{~cm}$ from the $\gamma$ entrance surface) as expected in MC. Energy resolution and time resolution for $55 \mathrm{MeV} \gamma$ will be measured in commissioning run in 2020, using $\gamma$ from $\pi^{0}$ generated with charge exchange reaction of $\pi^{-}$beam on liquid hydrogen target.

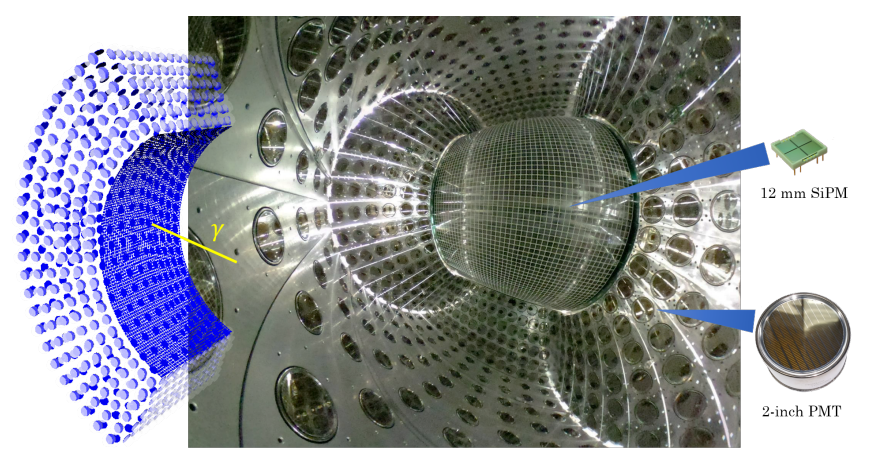

Figure 5: The liquid xenon detector 


\subsection{BG tagging detector}

BG tagging detector is a detector newly introduced in MEG II. It identifies the radiative muon decay (RMD, $\mu \rightarrow e v v \gamma$ ), which is one of the main sources of the background $\gamma$. RMD can be identified by detecting the low energy positron which drifts towards upstream or downstream of the beam axis under the magnetic field.

The detector consists of plastic scintillators for time measurement and LYSO crystals for energy measurement. It is placed $\sim 140 \mathrm{~cm}$ downstream of the muon stopping target and covers the region of $\pm 10 \mathrm{~cm}$ from the beam axis. The detector is already commissioned with a high-intensity muon beam, and the RMD identification capability was successfully demonstrated.

\subsection{Trigger and DAQ}

The waveform of all detectors is read out by the DRS chip developed at PSI with a sampling speed of a few GSPS. The WaveDREAM board is newly developed for MEG II. It contains 16 channels for waveform readout, with amplifier, shaper and bias voltage supply for SiPMs. The integrated trigger system can efficiently select the signal event by online $\gamma$ energy reconstruction, time coincidence and back-to-back requirement.

The prototype of the DAQ system with a limited number of readout channels has been tested in the commissioning runs. Full readout electronics is expected to be ready in early 2021.

\section{Status and prospect}

The construction of all the detectors is finished. For the TC and the background tagging detector, excellent performance has been already proven in the commissioning runs.

Some damaged wires were found in the $\mathrm{CDCH}$. These wires and other potentially weak wires have been successfully removed. The $\mathrm{CDCH}$ will be ready for the physics run once the stable operation under a high-intensity beam is established. In liquid xenon detector, photon detection efficiency of SiPMs was found to be reduced by $9 \%$ in 160 hours of muon beam run. A possible reason for this degradation is surface damage by scintillation light. However, this is not a critical problem because annealing of the SiPM is found to be possible for the recovery of the efficiency.

Further commissioning run of both detectors will continue in 2020 and 2021, followed by the physics runs after the completion of the detector performance study.

\section{Summary}

Lepton flavor violating decay of muon is a good probe of new physics beyond the Standard Model. Several next generation muon experiments are expected to start in five years. The MEG II experiment is aiming to search $\mu \rightarrow e \gamma$ with 10 times better sensitivity than MEG. The upgraded detectors are already constructed, and some of the detectors have shown excellent performance in the commissioning runs. A pilot run with full readout electronics is expected to take place in 2021, followed by the physics run.

\section{References}

[1] A. M. Baldini et al., The design of the MEG II experiment, Eur. Phys. J. C (2018) 78:380 